Tropical Journal of Pharmaceutical Research, February 2010; 9 (1): 81-90

(C) Pharmacotherapy Group, Faculty of Pharmacy, University of Benin,

Benin City, 300001 Nigeria.

All rights reserved.

Review Article

Available online at http://www.tjpr.org

\title{
Validation Aspects of Water Treatment Systems for Pharmaceutical Products
}

\author{
Rakesh Pahwa ${ }^{1 *}$, Mona Piplani ${ }^{1}$, Prabodh C Sharma ${ }^{1}$ and Arun \\ Nanda ${ }^{2}$ \\ ${ }^{1}$ Institute of Pharmaceutical Sciences, Kurukshetra University, Kurukshetra-136119, ${ }^{2}$ Department of Pharmaceutical \\ Sciences, Maharshi Dayanand University, Rohtak-124001, India
}

\begin{abstract}
The goal of conducting validation is to demonstrate that a process, when operated within established limits, produces a product of consistent and specified quality with a high degree of assurance. Validation of water treatment systems is necessary to obtain water with all desired quality attributes. This also provides a framework to establish a total control over the process which monitors safety, efficacy and ultimately, the process outcomes. The present overview is an attempt to discuss various aspects of validation including different approaches, components of water treatment systems, equipment qualifications, phases of performance testing, documentation and post-validation monitoring.
\end{abstract}

Keywords: Validation; Water treatment systems; Quality attributes; Pharmaceutical products. 


\section{INTRODUCTION}

Water may be referred to as 'the elixir of life' This term is most apt to the pharmaceutical industry for which the quality of water is critical and a high priority. It is an essential ingredient of various pharmaceutical preparations and is also used to clean process equipment and hence, plays a pivotal role in pharmaceutical processings [1]. It is, therefore, important that water should meet set standards given in different official texts; furthermore, the water treatment system constantly provides the specified quality and quantity of water so as to ensure that there is no contamination of the product or equipment. United States Pharmacopoeia (USP) describes several grades of this raw material (i.e., water), based on various quality parameters such as conductivity, total organic carbon (TOC), microbiological values, and presence of contaminants including endotoxins, nitrates and heavy metals. Water must be continuously tested and should comply with well defined quality attributes [2].

In the last few years, there has been an intensive focus on validation methodologies in the pharmaceutical industry. Its real importance within a productive process is recognised in relation to a product's quality attributes such as purity, safety and effectiveness. The Food and Drug Administration (FDA) has published guidelines pertaining to the general principles of process validation and has also defined process validation "as an established documented evidence which provides a high degree of assurance that a specific process will consistently produce a product meeting its predetermined specifications and quality characteristics" [3].

The term, validation, is used generically to cover the entire spectrum of current good manufacturing practices (cGMP) and therefore, it is also an element of the quality assurance programme. Validation is legible through the documentation establishment and it guarantees a manufacturing process with assured product quality and homogeneity. Validation of water treatment systems is mandatory to obtain water with all the desired quality characteristics [4]. Pharmaceutical water production, storage and conveyance system should be validated because end-product testing alone is not a sufficient evidence to confirm with a high degree of assurance that the system operates as it is purported. In order to cater for the ever escalating quality needs of the pharmaceutical industry, water treatment systems, which are highly dynamic in nature, must be validated, closely monitored and controlled. This article is intended to discuss the effectiveness, consistency and reproducibility of a water treatment system along with its validation aspects.

\section{VALIDATION APPROACHES}

Validation studies constitute the quality assurance programmes associated with a particular product or process. They are designed to establish a total control over process outcomes. To accomplish this objective, there is a need for comprehensive documentation with all necessary information, guidelines and evidence. Depending on how and when the data are generated and utilised, validation is said to be prospective, retrospective or concurrent $[5,6]$.

\section{Prospective validation}

Prospective validation includes considerations that should be made before an entirely new product is to be introduced or when there is a change in the manufacturing process which may affect the product's quality characteristics, such as uniformity and identity. These studies are conducted, evaluated and the process and equipment systems are certified prior to initiation of routine production. 


\section{Concurrent validation}

Concurrent validation studies are based on information generated during actual implementation of the process. This is generally performed by carrying out inprocess testing and/or monitoring of critical operations during the course of actual normal process.

\section{Retrospective validation}

Retrospective validation of a process is carried out for a product already in distribution. This approach is based upon information accumulated from production, testing and control data. It involves trend analysis (using control chart, etc) of historical manufacturing and quality control data of the product. This type of validation approach may not be possible if there is inadequate or incomplete documentation and lack of consistency in manufacturing and test records. Data from different batches of the product produced using the same stable manufacturing process should be analysed to demonstrate the performance and consistency of the process over time.

\section{DESIGN CONSIDERATIONS}

Design considerations include general information regarding various components or parts of water treatment systems. The systems should be constructed using modular, off-the-shelf purification components to control cost and maximise validation efficiency, which ultimately complies with official/pharmacopoeial requirements [7]. These considerations should be designed properly so as to prevent microbial growth; also, materials of construction must be selected carefully. The various parts of water treatment systems that should be validated include the following:

\section{Piping}

Stainless steel is generally the material of choice for pipe network because of its non- reactive and corrosion-resistant nature along with ease of sanitisation. It can also be employed over a wide range of temperature. Plastic piping systems such as polypropylene and polyvinylidene fluoride can also be used, especially in certain biotechnology-based applications. Glass or polycarbonate resin may be used where transparency is needed. Polyvinyl chloride is generally utilised in ambient temperature systems but not when ozone is used as sterilant. All pipe joints should either utilise sanitary fittings or be butt-welded. Piping systems should be compatible with frequent sanitisation, thermal cycling and must be specified for drainability. They should be designed for reliability, pressure control, and avoidance of extractable contaminants [8-10].

\section{Holding tanks}

Stainless steel is the preferred material for water storage tanks and it must be verified for resistance to chemical sanitisers. Storage tanks vary in size, depending on need; however, 7500 - 15000 litres are commonly used sizes. Suitable insulation is required to store the water at high temperature which conserves energy as well. Storage tanks must be provided with a vent to tolerate fluctuations in water levels, and thus prevent any possibility of collapse. Vents should be fitted with a hydrophobic air filter to prevent microbial contamination from outside air. Vent filter must be located in a position on the holding tank from where it is readily accessible [11,12].

\section{Valves}

Commonly used valves in water treatment systems are gate, ball, butterfly and diaphragm types. For the removal of dissolved solids, diaphragm valves should preferably be used downstream from the main unit. In ozone process water systems, ozone-inert polymers, such as teflon, should only be employed as valve seats [11].

Trop J Pharm Res, February 2010; 9 (1): 83 


\section{Filters}

Filters are used at various sites of water treatment systems for the purpose of removing undissolved solids and bacterial contaminants. Granular or cartridge filters are used for pre-filtration. Filters, commonly used downstream from carbon beds, should have 10 - $50 \mu$ pore size while membrane filters used to remove bacteria should have a pore size of $0.2 \mu$. Control measures for the filter include pressure and low flow monitoring, back washing and replacing filter media. For maintaining the efficiency of water treatment systems and avoiding any sort of endotoxin contamination or bacterial growth, filters must be maintained properly. Design and operational issues that may impact performance of depth filters include channelling of filtering media, blockage from silt, microbial growth and filter media loss [9].

\section{Deionisers and reverse osmosis (RO) units}

Their primary function is to remove dissolved solids from feed water. Deionisers use ion exchange resins to remove charged particles. Resins must be periodically regenerated using strong caustic and acid solutions as they loose their ability to remove charged particles. Cationic resins are regenerated with either hydrochloric or sulphuric acid, which replace the captured positive ions with hydrogen ions. Anionic resins are generally regenerated with sodium or potassium hydroxide, which replace the captured negative ions with hydroxide ions. RO system includes an additional integrated pretreatment cartridge pack with activated carbon, a $0.5 \mu$ prefilter, and a calcium hardness sequestering compound. Sequestering agent is a solid, long chain polyphosphate that weakly binds calcium ions and minimises calcium carbonate precipitation. Combination of pretreatment protects the $\mathrm{RO}$ membrane from damage due to fouling from particulates, chlorine oxidation and formation of mineral scale on membrane surface. Periodic chemical sanitisation treatments should be carried out on RO units so as to keep a control on bacterial growth $[7,11]$.

\section{Carbon beds}

These are commonly used to remove organic chlorine compounds and low molecular weight carbon compounds dissolved in feed water. Required design features include selection of proper particle size, avoiding hydraulic channelling and assuring proper adsorption capacity. Carbon beds can act as a source of bacterial and endotoxin contamination when improperly maintained, as organic material generally gets concentrated and accumulated in beds, which then becomes breeding ground for bacteria. Carbon beds should be periodically heat-treated using steam to get rid of these bacteria [9].

\section{Ultraviolet (UV) lights}

Their prime function is to act as a surface disinfectant as well as for the removal of ozone from ozone process water systems. To be effective, the absorbed ultraviolet dose must be sufficient to kill microorganisms. UV energy penetrates the outer cell membrane, passes through the cell body and disrupts its DNA, preventing reproduction. The most commonly used wavelength for microbial reduction in pharmaceutical water treatment systems is $254 \mathrm{~nm}$. Special low pressure mercury vapor lamps produce UV radiation at $254 \mathrm{~nm}$, the optimal wavelength for disinfection and ozone destruction. These lights must be sized properly for required water flow and UV lamps should be monitored as well as replaced whenever necessary. UV dose is computed using three independent variables such as lamp intensity, residence time distribution and water transmittance. To ensure that UV dose (lamp output) is effective, each of these process variables should be properly measured. Validation of UV light systems are necessary to ensure that sufficient disinfection performance (dose) is achieved under given 
flow rate and water quality conditions $[11,13]$.

\section{Distillation stills}

Distillation equipment is used to remove nearly all impurities from water which includes sodium, hardening compounds such as calcium and magnesium, other dissolved solids (including iron and manganese), fluoride and nitrate. When operated properly, it effectively inactivates various microorganisms such as bacteria, viruses, protozoan cysts, etc. It can also remove many organic compounds, heavy metals, chlorine, chloramines and radionucleides. Selection of a distillation unit should be based on analysis of various parameters of water. Also, regular maintenance of the unit is a critical factor in maintaining its effectiveness [14].

\section{Ozone and heat sterilants}

Ozone is a very strong oxidising agent with powerful disinfecting properties and can be easily removed from water by exposure to UV light. It is an effective bactericidal, viricidal, fungicidal as well as sporicidal agent in water treatment systems. It directly attacks the outer surfaces of microorganism and destroys their cell walls and membranes. In contrast to other oxidants and disinfectants, the use of ozone results in far fewer toxic disinfection byproducts. Its production rate can be controlled by process parameters in order to avoid incorrect dosing and to ensure optimised efficiency. More commonly, heat is also used to control microbial growth in water treatment systems. While circulating through distribution loops, treated water is heated to $80{ }^{\circ} \mathrm{C}$ in the storage tanks [15]. Various components, along with their desired features and/or functions generally used in water treatment systems, are summarised in Table 1.

\section{Continuous flow, absence of dead legs and biofilms}

Sanitary systems for pharmaceutical waters imply the use of smooth surfaces with minimal connections. Connection points need to be smooth and should offer minimal space to harbour bacteria. No-flow or low-flow of water, particularly through water distribution piping can be a cause of microbial proliferation and development of a biofilm. Biofilm formation is affected by the surface topography and chemistry of piping system that varies for different materials of construction. Dead legs in sanitary piping systems are areas of piping, generally associated with an instrument sample valve connection point, where the branch section of piping exceeds a defined length. Dead legs in distribution piping provide a place for microbial growth, as they can cause no-flow conditions. Therefore, stagnant areas should be minimised wherever possible in both sanitary and non-sanitary systems. Recirculating loops keep the water circulating through carbon filters, resin beds, RO units and storage tanks which are often equipped with UV lights to curtail microbial count to as low a level as possible $[11,16]$.

\section{SPECIFICATIONS FOR VARIOUS GRADES OF WATER}

USP has stipulated the specifications and definitions of the various grades of water suitable for pharmaceutical use. It classifies pharmaceutical water as (i) purified water, used for the manufacture of oral preparations and other formulations, and (ii) water for injection (WFI), used for injectables, parenterals and intravenous fluids. USP also specifies that purified water and WFI must adhere to the Environmental Protection Agency's (EPA's) Part 141, National Interim Primary Drinking Water Regulations [2,11]. Table 2 presents the principal standards for purified water and water for injection.

\section{EQUIPMENT VALIDATION}

Validation of water treatment systems follows the same basic sequence as does the validation of other pharmaceutical manufacturing equipment. It is necessary that the system should be checked and certified as properly installed, equipped and properly 
Table 1: Summary of components employed in water treatment systems and their desired features / functions

\section{Component}

Pipings

Holding Tanks

Valves

Filters

Deionizers and Reverse Osmosis

Carbon Beds

UV Lights

Distillation Still

Ozone and Heat Sterilants

\section{Desired features/functions}

Selected material: stainless steel; should be designed for reliability, pressure control, nullifying presence of extractable contaminants.

Optimal size/capacity: 2000-4000 gallons,

Hydrophobic air filters: restrict entrance of microbes in tanks.

Generally used types: Gate, Ball, Butterfly and Diaphragm.

Removes undissolved solids and bacterial contaminants,

Control measures: Pressure and low flow monitoring, back washing, sanitizing, replacing filter media etc.

Removes dissolved solids, resins must be periodically regenerated.

Removes organic chlorine compounds and low molecular weight carbon compounds, required design features: selection of proper particle size, avoidance of hydraulic channelling etc.

Biocidal wavelength: $254 \mathrm{~nm}$; UV dose variables: lamp intensity, residence time distribution and water transmittance should be properly measured

Deactivates bacterial endotoxins and removes dissolved solids not otherwise removed by $\mathrm{RO}$ units and deionizers.

Strong oxidizing agent, effective at low concentration. Both are used as biocidal.

Table 2: Specified limits for purified water and water for injection

\begin{tabular}{lll}
\hline Parameter & Purified water & Water for injection \\
\hline Conductivity & $<1.3 \mu \mathrm{s} / \mathrm{cm}$ at $25^{\circ} \mathrm{C}$ & $<1.3 \mu \mathrm{s} / \mathrm{cm}$ at $25^{\circ} \mathrm{C}$ \\
$\mathrm{pH} 5.0-7.0$ & $5.0-7.0$ & \\
Bacteria & $<100 \mathrm{cfu}^{*} / \mathrm{ml}$ & $<10 \mathrm{cfu} / 100 \mathrm{ml}$ \\
Total organic carbon & $<500 \mathrm{ppb}^{* *}$ & $<500 \mathrm{ppb}$ \\
Endotoxins & $\mathrm{N} / \mathrm{A}$ & $<0.25 \mathrm{EU}^{* * *} / \mathrm{ml}$ \\
\hline
\end{tabular}

$c f u^{*}=$ colony forming units; $p p b^{* *}=$ parts per billions; $E U^{\star * *}=$ endotoxin units 
functioning as per its design [11,17]. Equipment validation has the following steps:

\section{Installation qualification (IQ)}

It is the process which ensures and proves that the system has been supplied and installed correctly, and meets the predetermined specifications of the users. It usually involves the generation of an IQ protocol, a test and inspection plan for the system. All installation parameters must be documented and certified prior to operational qualification of the system. For an IQ of water treatment system, the following would be typical key elements [11]:

- $\quad$ Utilities requiring verifications includes electricity, compressed air, steam and feed water. Each should be checked properly at the time of installation of equipment for water treatment systems.

- $\quad$ Calibration of all process controlling instruments according to written procedures and certification that they meet the specified tolerance limits for accuracy, precision, and also in terms of selectivity or specificity.

- Verify documentation on system design specifications including materials data and calibration certificates.

\section{Operational qualification (OQ)}

This consists of tests and inspections to verify that the equipment, system alerts and controls are operating reliably. It is a documented verification that the system or subsystem performs as intended throughout all anticipated operating ranges. This process not only corrects the operation of a system, but also assesses the adequacy of Standard Operating Procedures (SOPs). For an $\mathrm{OQ}$ of water treatment system, the following key parameters would be typical [11]:

- Testing of heat exchangers and stills at low and high ends of the ranges.

- Verification of valves, controllers and flow rates.

- SOPs should be written to describe start up, shut down and sanitisation procedures.

- Sanitisation of holding tanks and distribution parts of water treatment systems including periodic regeneration of resin beds.

- Evaluating pumps for proper functioning along with the replacement of any defective valves or seals.

\section{Performance qualification (PQ)}

After each major component of the water treatment system has been operationally verified, the next step is performance qualification. This phase includes various tests designed to verify the satisfactory performance of the system. It should be carried out using real process materials and tests intended to demonstrate accurate and reliable performance over the full range of expected operating conditions. $P Q$ is the stage which documents that the product quality continuously and consistently meets the required specifications. The major objective of this step is to demonstrate that the system produces and maintains recirculating water, which meets the compendial requirements for purified water over a suitable time period $[11,17]$.

\section{TESTING PHASES IN VALIDATION}

To complete the validation of water treatment systems, it requires as long as one year because of operating problems, equipment failures and maintenance errors expected to occur during the validation period. Also, it takes one complete year to determine the seasonal variation in the microbiology of feed water as well as to demonstrate the effectiveness of system sanitisation procedures against resident organisms [11]. Successful and complete validation programme of water treatment systems can 
also be categorised into various phases:

\section{Phase I testing}

This preliminary and investigational phase of validation requires a test period of $2-4$ weeks for monitoring the system intensively [18]. In this phase, initially the system is operated continuously without failure or performance deviation. Frequent and extensive sampling along with testing at numerous locations of operation is also carried out. Chemical and microbiological studies are performed on the samples in accordance with a defined plan [17]. This phase also involves the development of appropriate operating ranges along with the finalisation of cleaning, sanitising and maintenance procedures. At the end of this phase, systems are simulated to perform under stress conditions such as start-up after power failure or emergency shut downs. On the other hand, the system is also tested under normal maintenance situations such as start-up after resin regenerations, filter changes, etc. If the system uses ozone, a failure of ozone generators should be simulated. Lastly, SOPs for the water systems should be developed.

\section{Phase II testing}

It is a continuation step following phase I and generally includes the same sampling scheme as in phase I. It should further facilitate intensive monitoring of the system for a test period of 2 - 4 weeks. Deployment of all the refined SOPs is done in this phase after satisfactory completion of phase I. Water can be used for manufacturing purposes during this phase. This step demonstrates that the system is under control within established ranges. Testing at this phase also ensures consistent production and delivery of water of required quantity and quality when the system is operated in accordance with SOPs [18].

\section{Phase III testing}

It is a confirmatory step in which frequency and number of sampling locations are less than in phase I and II. This phase demonstrates that the system exhibits extended reliable performance and is under control over a long period of time. It requires a span of around one year after the satisfactory completion of phase II. Water can be used for manufacturing purposes during this phase. Seasonal variations of the feed water are also evaluated in this phase [18]. This final phase should be initiated only after the requirements mentioned in the protocols for phase I and II testing have been satisfied. Moreover in this phase, complete chemical and microbiological analysis must be performed and results should be presented graphically using various computer simulations. Complete validation report must be written, reviewed and approved according to the firm's procedures. Validation project can not be considered accomplished until the final reports get approved by appropriate authorities. Maintenance and operating personnel must also be trained to do their job flawlessly and may be required to demonstrate proficiency.

\section{POST-VALIDATION MONITORING}

Post-validation monitoring consists primarily of routine checking of water treatment systems against the validated cycle, routine sampling and ongoing equipment maintenance. Each phase must be monitored to ensure that all the desired conditions were set as specified. These checks should be documented in the processing records. The requirements to perform monitoring should be a detailed written procedure referenced in the validation protocol. Deviations from defined processing conditions must be documented, investigated and assessed for compliance with the protocol. In order to ensure that the equipment and support systems function consistently within the validation protocol specifications, there should be a written programme for the ongoing maintenance of 
each part of equipment defined in the protocol. The maintenance program should furnish details of the items to be checked and the frequency of maintenance and calibration of monitoring devices.

The post-validation phase is very crucial and essential for the maintenance of a system in a state of control by keeping the equipment and process within acceptable ranges established during the validation programme. Information obtained from periodic sampling and testing of water from various distribution units must comply with the specified validation protocols $[6,11]$.

\section{CONCLUSION}

Water treatment systems must be operated within regulatory guidelines as with pharmaceutical production facilities. To validate these systems, there must be documented evidence that the system is operating consistently and according to the desired specifications. Validation is a tool for total quality management and it is necessary for process optimisation, safety, efficacy and assurance of quality. Such validation protocols also fulfill regulatory requirements and provide good business sense. Successful accomplishment of validation is ensured by various testing phases. Usually, a three-phase testing approach is recommended over an extended period to prove reliability and robustness of the system for producing water of specified quality with a high degree of assurance.

\section{ACKNOWLEDGEMENT}

Professor Om Prakash, Director, Institute of Pharmaceutical Sciences, Kurukshetra University, Kurukshetra-136119, India, is duly acknowledged for providing facilities for this study.

\section{REFERENCES}

1. Hultqvist A. Practical guidelines for qualifying purified water systems. Pharm Technol Europe
2007; 19(12). http://www.ptemag.com/pharmte cheurope/Validation/ArticleSta ndard/Article/de tail/480191. Accessed 24 May 2009

2. The United States Pharmacopoeia, USP XXII/The National Formulary, NF XVII, 1990, United States Pharmacopeial Convention, 12601 Twinbrook Parkway, Rockville.

3. Food and Drug Administration, Guideline on general principles of process validation. FDA, Rockville, MD 1984.

4. PMA Deionized Water Committee, Validation and control concepts for water treatment systems. Pharm Technol 1985; 9(11):50-56.

5. Nash RA. Validation of pharmaceutical processes. In: Swarbrick J, Boylan JC (Eds). Encyclopedia of Pharmaceutical Technology. $2^{\text {nd }}$ ed. New York: Marcel Dekker, 2002; pp 2917-2931.

6. Pahwa $R$, Khatri $S$, Rathour A, Lamba HS. Validation of moist heat sterilization cycles. J Sci Pharm 2004; 5(4):125-130.

7. Tunner J, Katsoulis G, Denoncourt J, Murphy $S$. Design, qualification and performance of a cost-effective water purification system for a GMP pilot plant. Pharm Engg 2006; 26(4):1-8.

8. Sixsmith $T$, Jackson J. How piping materials for the pharmaceutical industry compare to each other. Ultrapure Water 1999; 16(4):53-59.

9. Gupta RM, Vishweshwar S, Bhingare CL, Trivedi N. Design qualifications for water purification system. Express Pharma Pulse 2002. http://www.expresspharmaonline.com /20020704/technology1.shtml. Accessed 29 May 2009

10. Baird A, Kirsten S, Ralph W. Comparison of high purity water for microelectronic and biopharmaceutical facilities. Pharm Engg 2001; 21(5):34-46.

11. Johnson WM. Validation of water systems for sterile and nonsterile products. In: Berry IR. Nash RA. (Eds). Pharmaceutical Process Validation. $2^{\text {nd }}$ ed. Revised and Expanded, New York: Marcel Dekker Inc, 1993; pp 299-317.

12. Guide to inspections of high purity water systems (US FDA, 1993). http://www.bcg-usa.com/r egulatory/docs/1993/FDA199307E.pdf. Accessed 2 June 2009.

13. Sarchese M. UV-Moving into the mainstream. Water Quality Products 2006; 11(10). http://www.wqpmag.com:80/popup app/index. cfm?fuseaction. Accessed 2 June 2009.

14. Dvorak BI, Skipton SO. Drinking water treatment: Distillation. 2008. http://www.ianrpubs.unl.edu/ epublic/live/g1493/build/g1493.pdf. Accessed 25 May 2009.

15. Nebel $C$, Nebel T. Ozone, the process water sterilant. Pharm Manuf 1984; 4(2):16-23.

16. Gillis RJ, Gillis JR. A comparative study of bacterial attachment to high purity water system surfaces. Ultrapure Water 1996; 13(6):27-36.

17. Raghunandanan $R$. Validation aspects of solid dosage forms. Pharma Times 2009; 41(4):1518.

Trop J Pharm Res, February 2010; 9 (1): 89 
Pahwa et al

18. Supplementary training modules on GMP. WHO technical report series, No. 937, 2006, http://apps.who.int/prequal/trainingresources/p
q_pres/gmptrainsup/mt/Validation_Part2.ppt Accessed 10 June 2009. 